\title{
Trajectoires d'une ruralité recomposée en Europe centrale
}

Rural Trajectories in Central Europe

\section{Guillaume Lacquement, Marie-Claude Maurel et Jean-Claude Raynal}

\section{(2) OpenEdition}

Journals

\section{Édition électronique}

URL : http://journals.openedition.org/economierurale/3234

DOI : 10.4000/economierurale.3234

ISSN : 2105-2581

Éditeur

Société Française d'Économie Rurale (SFER)

\section{Édition imprimée}

Date de publication : 21 octobre 2011

Pagination : 39-53

ISSN : 0013-0559

\section{Référence électronique}

Guillaume Lacquement, Marie-Claude Maurel et Jean-Claude Raynal, «Trajectoires d'une ruralité recomposée en Europe centrale », Économie rurale [En ligne], 325-326 | septembre-décembre 2011, mis en ligne le 17 octobre 2013, consulté le 24 avril 2019. URL : http://journals.openedition.org/ economierurale/3234; DOI : 10.4000/economierurale.3234 


\title{
Trajectoires d'une ruralité recomposée en Europe centrale
}

\author{
Guillaume LACQUEMENT • Université de Perpignan, FRE 3027 du CNRS \\ Marie-Claude MAUREL • EHESS, UMR 8083 EHESS-CNRS \\ Jean-Claude RAYNAL • Ingénieur d'études à l'EHESS
}

P enser le changement social et la transformation spatiale dans leur cheminement solidaire, traiter des rapports entre la reconfiguration des territoires et la mutation des sociétés qui les construisent et leur donnent sens, telle est la perspective adoptée. La recomposition des espaces ruraux en Europe centrale procède d'une rupture de système politique et social qui a ouvert un temps de transformation de la ruralité, étendu sur deux décennies. Par son ampleur et sa rapidité, cette mutation est inédite dans l'histoire des sociétés rurales européennes (Maurel, 1994). Ses effets ne peuvent être comparés qu'aux bouleversements structurels introduits par les réformes agraires de la première moitié du $\mathrm{xx}^{\mathrm{e}}$ siècle ou par la collectivisation des années cinquante. D'une certaine manière, les transformations récentes leur ont fait écho. $\mathrm{La}$ reconfiguration des structures spatiales apparaît soumise à des inerties et à des décalages qui tiennent compte de la durée propre aux formes agraires héritées et du temps d'émergence de nouvelles formes spatiales. Les trajectoires de recomposition des territoires ruraux procèdent de la combinaison spécifique d'un effet de temps et d'un effet de lieu qui renvoient aux durées variables des formes spatiales. La notion de trajectoire est ici entendue comme un instrument conceptuel permettant d'intégrer la dimension temporelle dans l'étude du processus de changement spatial, de considérer les notions de simultanéité, de succession d'évènements et de durée des phénomènes dans la lecture des mécanismes de transformation qui affectent l'organisation de l'espace.
Les approches théoriques de la transformation de système se distinguent par la manière dont elles conceptualisent le changement, soit par rapport à un état final souhaité (paradigme de la transition), soit dans une perspective attentive aux temps propres des institutions et des structures (paradigme de la path dependence). Les changements peuvent être appréhendés selon ces deux visions concurrentes, l'une mettant l'accent sur la rupture entre le passé et le présent, l'autre sur le caractère évolutionnaire et organique de la transformation, et sur la manière dont s'élabore une symbiose complexe entre éléments anciens et nouveaux. C'est cette dernière conception qui sous-tend la démarche de recherche.

\section{Interpréter la transformation postsocialiste}

\section{Spécificité de la ruralité centre-européenne}

Une première approche postule l'existence d'une spécificité des territoires ruraux centre-européens par rapport aux évolutions enregistrées dans les pays d'Europe occidentale. L'hypothèse centrale est celle d'une histoire radicalement différente dans la longue durée comme dans le temps court des transformations contemporaines, avant, pendant et après la période socialiste. La prise en compte du temps long éclaire certaines des spécificités de ce que les historiens ont appelé l'Europe des grands domaines. Mis en place au début des temps modernes, le dualisme de l'Europe agraire, le système des grands domaines, à l'Est, celui des seigneuries et des communautés 
paysannes, à l'Ouest, a durablement marqué les rapports sociaux et a déterminé la situation socio-économique de la paysannerie au sein de chacun de ces ensembles (Rösener, 1994, Szúcs, 1985) ${ }^{1}$. Tandis qu'en Europe occidentale se développait un modèle paysan, ancêtre de l'exploitation familiale moderne contemporaine, une telle évolution se trouvait bloquée par le système de la grande propriété foncière en Europe orientale. À partir des années vingt du $\mathrm{XX}^{\mathrm{e}}$ siècle, des réformes agraires ont tenté de généraliser l'idéal d'une paysannerie indépendante et propriétaire de ses terres, mais cette évolution a été interrompue par la collectivisation imposée à l'Europe centrale et orientale, à partir des années cinquante. Selon des modalités variables, avec des hésitations, voire des retours en arrière, comme en Pologne où une petite propriété paysanne a résisté, ce système a perduré jusqu'en 1989 (Maurel, Halamska, Lamarche, 2004). En fonction des voies nationales de socialisation de l'agriculture, le modèle initial a subi diverses altérations, susceptibles de laisser des traces dans les structures comme dans les rapports sociaux et les comportements. Les activités du secteur primaire (agriculture, forêts, pêche) ont assuré la base économique dans les campagnes tandis que les fonctions de services, généralement intégrées au sein des exploitations collectives, sont restées d'importance limitée. L'exode rural s'est intensifié au bénéfice des centres industriels sans qu'un processus de périurbanisation se développe à la périphérie des centres urbains. La pluriactivité en milieu rural s'est localement incarnée dans la figure de l'ouvrierpaysan se déplaçant du village vers le centre industriel.

1. La ligne de partage historique entre les deux grands types de systèmes agraires coïncidait à peu près avec le cours de l’Elbe, Szúcs (1985).
À l'appui d'une vision de la spécificité du rural centre-européen, un certain nombre de données factuelles peuvent être avancées : les densités humaines élevées des espaces ruraux centre-européens, produit de l'accumulation des excédents démographiques sur place et d'un exode rural plus tardif ; le contenu socioéconomique des activités en milieu rural marqué par le poids dominant de l'emploi agricole, en dépit du délestage récent. La sortie du système collectiviste dans un contexte de crise et de déprise économique est venue en quelque sorte confirmer ce destin particulier. La transformation post-collectiviste aurait ainsi préservé de la dualité des formes sociales de production : petite agriculture de subsistance versus grande exploitation agricole (Small, 2003 ; Streith, 2005) $)^{2}$. Plusieurs travaux ont mis l'accent sur le fait que l'évolution récente contribue à accentuer les caractères propres aux sociétés rurales d'Europe centrale. Ils avancent les termes de « campagne post-socialiste » (Swain, 1999, de « question agraire post-socialiste » (Hann, 2003), évoquent la figure d'un « paysan post-socialiste » se définissant par sa filiation socialiste (Leonard et Kaneff, 2002). Pour ces auteurs, le devenir du «paysan postsocialiste » ne peut être compris sans référence aux évènements passés et aux contextes idéologiques particuliers dans lesquels ces figures sociales se sont formées. Cette irréductible spécificité rendrait problématique la convergence avec les anciens pays membres au sein de l'Union européenne.

\section{Adaptation et convergence}

Une autre interprétation considère les différences entre les campagnes de l'Europe occidentale et celles de l'Europe centrale avant tout comme l'expression d'un décalage temporel, d'une forme de développe-

2. Small (2003) n'hésite pas à voir dans la persistance de la petite exploitation une opportunité, voire un modèle de développement durable. 
ment retardé. D’origine historique, ce gradient de modernité décroissante d'ouest en est, procèderait de la diffusion plus précoce des innovations et du progrès technique et économique à l'ouest de l'Europe qu'à l'est (Grandberg, Kovach, Tovey, 2001). Un temps, différée ou dévoyée par l'application du modèle collectiviste, la modernisation de l'agriculture et de la campagne reprendrait son cours depuis 1990 (Rey, 1996). Le retour à l'économie de marché engagerait une logique de rattrapage que l'intégration européenne favoriserait. Cette approche théorique ouvre sur une perspective de convergence, fruit de la réussite de la transformation post-collectiviste. Le clivage historique majeur affectant les fondements agraires de l'Europe pourrait être surmonté grâce au transfert institutionnel opéré sous l'égide de l'Union européenne (UE). Ce transfert porte sur l'introduction de l'entreprise agricole privée à l'issue de la décollectivisation, ainsi que sur l'adoption des principes de l'autonomie locale, au début de la décennie 1990. Il emprunte aujourd'hui la voie des politiques de développement rural intégré (Maurel, 2008). La mise en œuvre des politiques européennes se présente comme un acte de transposition de dispositifs institutionnels et de politiques publiques conçus et expérimentés par les anciens États membres. Il s'incarne dans l'adoption de la PAC et de «l'acquis communautaire », l'introduction d'initiatives communautaires de promotion du développement local telles que LEADER (Kovach, 2000 ; Kovach et $\mathrm{Ku}$ čerova, 2006) ainsi que, plus récemment, dans la mise en œuvre de politiques environnementales (Gorlach et Kovach, 2006 ; Marty, Devaux, 2009).

La thèse de l'adaptation aux normes européennes conduit à s'interroger sur les modes de réception et d'apprentissage de ces modèles par les nouveaux Etats membres, en particulier par les sociétés rurales centreeuropéennes (Hirschhausen et Lacquement, 2008). Comment adoptent-elles les cadres institutionnels de la démocratie et de l'économie de marché ? À quel rythme et dans quelle mesure ces emprunts modifient-ils leurs comportements et leurs pratiques ? Y a-t-il rupture avec les systèmes de valeurs du passé ou transcription active de celles-ci dans les nouveaux cadres institutionnels ? Quelles stratégies de développement local les sociétés rurales sont-elles disposées à mettre en œuvre?

Les deux thèses interprétatives, que l'on vient de brièvement évoquer ci-dessus, ne simplifient-elles pas des processus de recomposition plus complexes à l'échelle des territoires ruraux?

\section{Le changement fonctionnel des espaces ruraux}

Si les politiques d'intégration au sein de l'UE ont fait le pari de la convergence des évolutions, le changement fonctionnel demeure tributaire d'un héritage socialiste qui peut constituer un obstacle à l'adaptation de l'économie rurale et à sa diversification. S'ils partagent des similarités de destin et des legs structurels, les espaces ruraux centre-européens traversent à présent une phase de re-différenciation. On se bornera ici à dresser la typologie des évolutions démographiques ainsi que celle des formes de diversification des activités socioéconomiques pour décrire les trajectoires du changement fonctionnel en cours ${ }^{3}$. Prenant appui sur la transformation lente des valeurs d'usage de l'espace rural et la recomposition des figures de la ruralité, le changement est diversement avancé selon les lieux.

\footnotetext{
3. Les matériaux empiriques qui ont été recueillis et traités (données statistiques au niveau NUTS 4, enquêtes répétées de terrain) invitent à une lecture nuancée des différenciations géographiques dans une démarche résolument comparative.
} 


\section{Des évolutions démographiques contrastées}

Déprise, dépeuplement et périurbanisation

La variation démographique, l'évolution des comportements migratoires et naturels des populations soulignent les inflexions de trajectoires révélatrices des changements socioéconomiques vécus par les espaces ruraux centre-européens. Les structures démographiques se transforment sous l'effet des déstructurations du tissu socioéconomique liées au passage à l'économie de marché. Les données révèlent le déclin de la ruralité dans un contexte généralisé de perte de vitalité démographique. Les évolutions sont tributaires des formes du peuplement rural et des caractéristiques du maillage urbain.

La typologie présentée s'appuie sur des données strictement comparables entre elles, c'est-à-dire les composantes démographiques (croît naturel, solde migratoire et variation de la population 1995-2001). La dynamique démographique des populations rurales repose sur le jeu conjoint du croît naturel et des échanges migratoires. À partir des valeurs de ces deux composantes, la carte du bilan démographique permet de caractériser les types de trajectoire des espaces ruraux (carte 1, cf. la légende). Les trois premiers groupes connaissent une croissance plus ou moins affirmée de leur population, les trois suivants enregistrent des pertes conduisant au dépeuplement rural.

- Le premier type correspond à une phase de dynamisme démographique. Cette croissance résulte de la conjonction d'un stimulant exogène, le solde migratoire toujours positif, même s'il connaît un certain tassement, et d'une dynamique endogène illustrée par un solde naturel également positif. Les couronnes des grandes villes polonaises (Cracovie, Poznań, Gdańsk, Szczecin, Wrocław) mais aussi des villes de moindre taille (Kielce, Radom, Konin, Olsztyn) attestent de la diffusion périurbaine. La première couronne de Bratislava, les districts proches de Košice et de Prešov en Slovaquie sont dans ce même cas de figure. Ces bassins d'emploi urbains exercent un pouvoir attractif sur des populations jeunes comme en témoignent la pyramide des âges et les comportements naturels. De manière plus limitée, le retour à un croît naturel positif dans le cas de quelques districts (en Bohême méridionale), son maintien dans d'autres, indiquent une reprise de la croissance endogène de la population rurale.

- Le deuxième type correspond à une phase de repeuplement, sous l'influence des apports migratoires. L'ampleur de ceux-ci permet un accroissement de la population totale et favorise un rééquilibrage de la pyramide des âges et la réduction du déficit naturel. Ceci concerne surtout les espaces à proximité des grandes agglomérations (Berlin, Varsovie, Budapest, Vilnius, Prague) où se développent de nouvelles activités tertiaires et secondaires, et un processus soutenu d'installation résidentielle périurbaine. Il peut s'agir également d'espaces proches de métropoles régionales, au cœur de bassins d'emplois dynamiques (Debrecen, Győr en Hongrie, Brno et České Budejovice en République tchèque, Klaipéda, Kaunas en Lituanie). L'attractivité migratoire y compense largement le déficit naturel, exprimant un processus actif de périurbanisation. L'installation de nouveaux résidents et le rajeunissement démographique y vont de pair.

- Le troisième type est marqué par une variation faiblement positive de la population totale, l'excédent naturel venant compenser le déficit migratoire. La structure par âge conserve une proportion de jeunes constituant un réservoir potentiel. Cette trajectoire démographique ne se rencontre que dans quelques districts de Pologne (Cachoubie, Basses Carpates), ainsi que dans les espaces ruraux de Slovaquie centrale et orientale qui préservent leur potentiel démographique.

- Le quatrième type correspond à une phase démographique transitoire, dans son ensemble. Ces espaces se distinguent par un 
RECHERCHES

Guillaume LACQUEMENT, Marie-Claude MAUREL, Jean-Claude RAYNAL

Carte $n^{\circ} 1$. Bilan des comportements démographiques

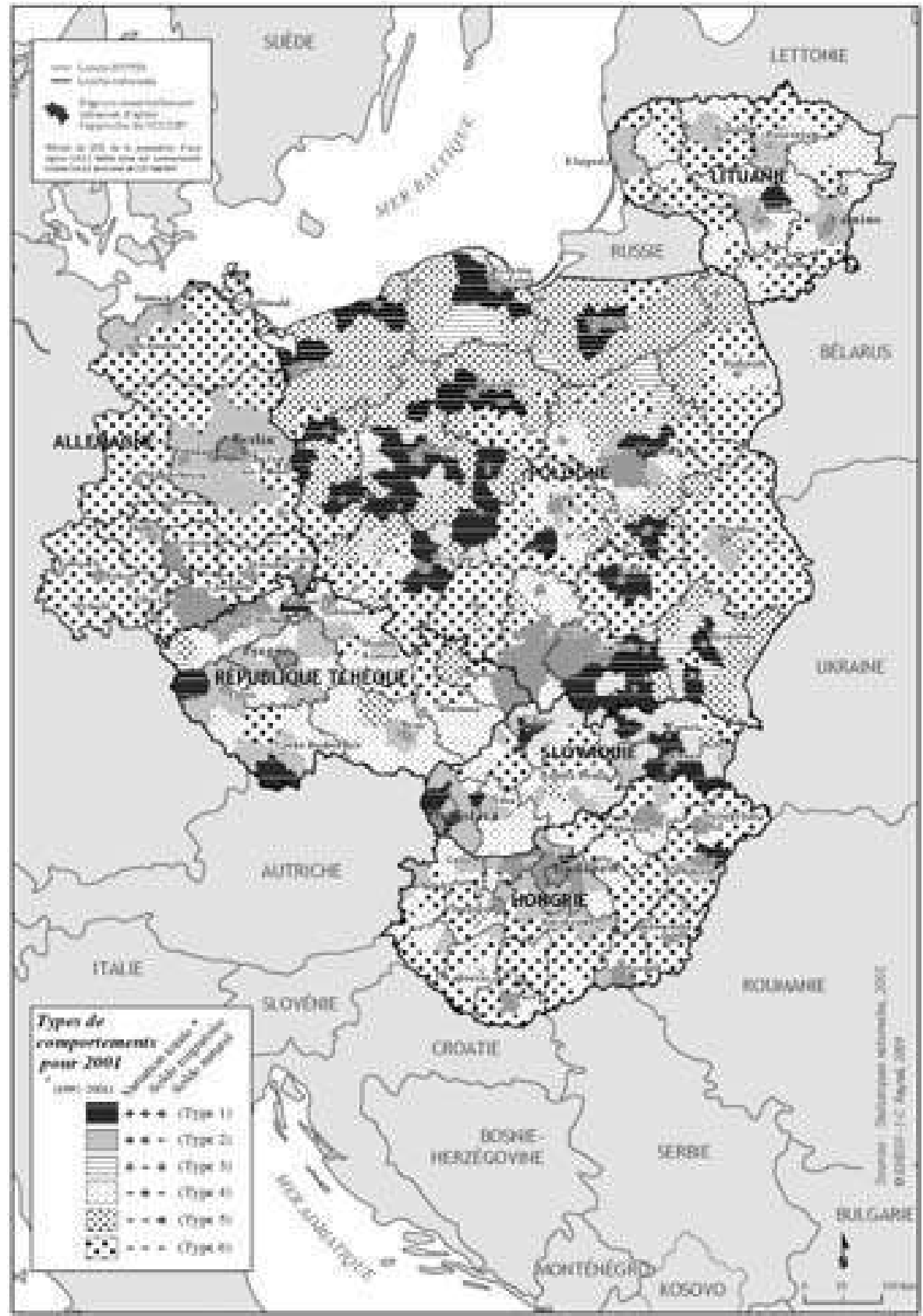

tassement progressif de l'exode rural sous l'exode rural antérieur. De nouvelles l'effet d'une inversion des flux migratoires. formes de mobilité résidentielle commenLe solde migratoire est positif. Il tend à cent à s'affirmer. Au terme de cette phase contrebalancer le déficit naturel consécutif de transition, l'équilibre entre les deux au vieillissement démographique lié à composantes démographiques favorise une 
stabilisation de la population rurale. Cette situation qui tend à se généraliser en Bohême et plus largement en Moravie, n'affecte ailleurs que des espaces plus limités.

\section{- Le cinquième type est caractérisé par une} diminution de la population, le déficit migratoire l'emportant sur le croît naturel. Il correspond à une situation de réservoir démographique en baisse. Cela concerne les territoires ruraux du nord et de l'ouest de la Pologne, de la Basse Silésie à la Mazurie, frappés par les restructurations de l'ancien secteur agricole d'État. Le déficit migratoire affecte les populations les plus jeunes touchées par le chômage et laisse présager une prochaine inversion du croît naturel.

- Le dernier type regroupe les espaces en déclin démographique résultant d'une perte de vitalité démographique (solde migratoire négatif et déficit naturel avec un vieillissement accusé). Les espaces concernés sont très étendus. En Allemagne et en Hongrie, plus de $60 \%$ des régions rurales appartiennent à la catégorie des espaces démographiquement déprimés. Le départ de la main-d'œuvre jeune vers les marchés de l'emploi urbain ou vers l'étranger va de pair avec un vieillissement de la population. Pratiquement toutes les campagnes d'Allemagne orientale sont touchées par cette récession démographique. Les déficits naturel et migratoire se creusent dans les plaines agricoles du centre et du nord du pays, tandis que les régions de moyenne montagne de Saxe et de Thuringe peinent à retenir leurs forces vives en raison de la désindustrialisation. En Pologne, les régions de déclin forment un vaste ensemble, depuis le piémont des Sudètes jusqu'aux confins orientaux, en Podlasie et dans la région de Lublin. C'est la Pologne des populations rurales vieillies. En Lituanie, les espaces ruraux périphériques sont principalement concernés.

Les effets territoriaux de la transformation post-socialiste se traduisent par des dynamiques sociodémographiques différenciées, la déprise et la perte de vitalité démographique de larges espaces, contrastant avec les situations de croissance et d'attractivité de territoires plus restreints et généralement sous influence urbaine. Loin de s'effacer, les contrastes ont tendance à se renforcer sous l'effet de changements structurels radicaux liés à la généralisation de l'économie de marché.

\section{Diversification socio-économique et contrastes spatiaux}

Une deuxième typologie de synthèse, effectuée à partir d'une sélection d'indicateurs pertinents, vient illustrer la diversité des trajectoires de diversification fonctionnelle des territoires ruraux. La catégorisation selon les fonctions économiques est une approche classique en géographie, elle a notamment été illustrée par les travaux de géographes polonais (Bański et Stola, 2002) et hongrois (Csatári, 2005), plus récemment par une analyse des bases économiques de l'espace rural tchèque (Chevalier, 2008). Le retournement des logiques de gestion des espaces ruraux a conduit à des adaptations structurelles qui, à l'échelle régionale, ont dessiné des trajectoires différenciées ${ }^{4}$.

4. Le processus de diversification des fonctions économiques et sociales peut être saisi à partir d'indicateurs statistiques collectés aux niveaux UAL1 et UAL2 de la nomenclature statistique européenne. Le travail d'analyse s'appuie sur un traitement statistique multivarié qui pointe la situation de la diversification socioéconomique des campagnes une décennie après l'introduction de l'économie de marché (2001). Les indicateurs retenus mesurent la mobilité de la maind'œuvre entre les secteurs de l'économie (taux d'actifs dans le secteur secondaire et dans le secteur tertiaire, part des exploitations agricoles nonmarchandes). Ils proposent également d'évaluer l'investissement des populations locales dans la recherche de nouvelles ressources économiques (en particulier dans le développement des activités touristiques (intensité touristique par nombre de nuitées) et dans le choix de modèles agricoles alternatifs (part des exploitations agricoles biologiques dans le total des exploitations). 
Ces dernières dépendent d'un faisceau de facteurs qui conjuguent leurs effets sur le processus de restructuration territoriale en cours $^{5}$. Les États ont progressivement formulé, puis mis en œuvre des politiques de développement rural, dont les dispositifs fondamentaux ont orienté le processus de sortie de crise par la diversification des activités économiques ${ }^{6}$. Référées au modèle ouest-européen d'aménagement rural, ces politiques ont déterminé et encadré des trajectoires de diversification, fondées sur le renouvellement des fonctions spatiales par la valorisation de ressources économiques nouvelles. Les évolutions socio-économiques dessinent des trajectoires différenciées, qui dépendent autant de l'héritage des structures spatiales de l'époque socialiste et des époques antérieures, que des formes contemporaines de l'implication des sociétés locales dans le processus de transformation.

Des trajectoires de spécialisation fonctionnelle se greffent sur des structures et des activités héritées (carte 2). Dans la plupart des régions concernées, le développement récent du tourisme procède d'une évolution économique et d'une structuration territoriale qui précèdent la transformation systémique de 1990 (type 1, spécialisation touristique). L'ouverture de l'économie ne fait qu'amplifier le volume des flux de fréquentation sur des espaces ruraux qui poursuivent leur spécialisation en lien avec une activité extravertie et

5. L'analyse statistique multivariée apporte un éclairage synthétique sur le jeu de ces facteurs et permet de dresser une typologie des trajectoires de diversification socio-économique, et de caractériser les formes de la structuration territoriale du rural centreeuropéen (carte 2).

6. La situation de crise, provoquée par le changement de système, se manifeste par le recul généralisé de l'emploi agricole découlant des restructurations, le repli de l'activité des bassins industriels fournissant des emplois aux paysans-ouvriers, le ralentissement des migrations vers les pôles urbains. dépendante des pôles émetteurs de clientèle.

Le maintien d'une tradition industrielle caractérise les campagnes ouvrières nées de l'implantation des grands complexes industriels à l'époque socialiste (type 2 , crise et reconversion des activités manufacturières). Dans un contexte de désindustrialisation, l'adaptation des systèmes productifs aux exigences des marchés se fait très difficilement. La structure territoriale héritée perdure en maintenant des formes de dépendance de la main-d'œuvre rurale à l'échelle de bassins d'emploi. Les reconversions s'opèrent au prix d'un délestage massif des emplois et de la relocalisation sélective des fabrications. Les nouvelles formes d'investissement qui dépendent le plus souvent de centres de décision extérieurs orientent alors les fonctions de ces espaces vers une logique de sous-traitance industrielle. Les capacités de reconversion semblent insuffisantes pour assurer le développement de ces espaces qui nécessiterait des dispositifs de soutien adaptés.

La transformation du tissu des petites et moyennes entreprises et l'adaptation des branches de production peuvent participer à une dynamique réelle de diversification des activités rurales. Des trajectoires de diversification concernent ponctuellement des régions où les indicateurs expriment l'émergence d'une multifonctionnalité rurale (type 3, formes diversifiées de pluriactivité rurale $)^{7}$. Les formes structurelles en sont apparemment diverses. Dans des territoires aux densités de peuplement variables, le changement fonctionnel introduit une diversification sans exclusive qui s'appuie sur un assemblage d'activités plurielles en partie héritées et qui bénéficie d'investissements exogènes relayés par des initiatives endogènes, susceptibles de ralentir les processus récessifs de déprise

7. Tels que le taux d'actifs dans les secteurs secondaire et tertiaire, la part relative de l'agriculture marchande, la fréquentation touristique, etc. 
Europe centrale : une ruralité recomposée

Carte $n^{\circ} 2$. Typologie des trajectoires de diversification socio-économique

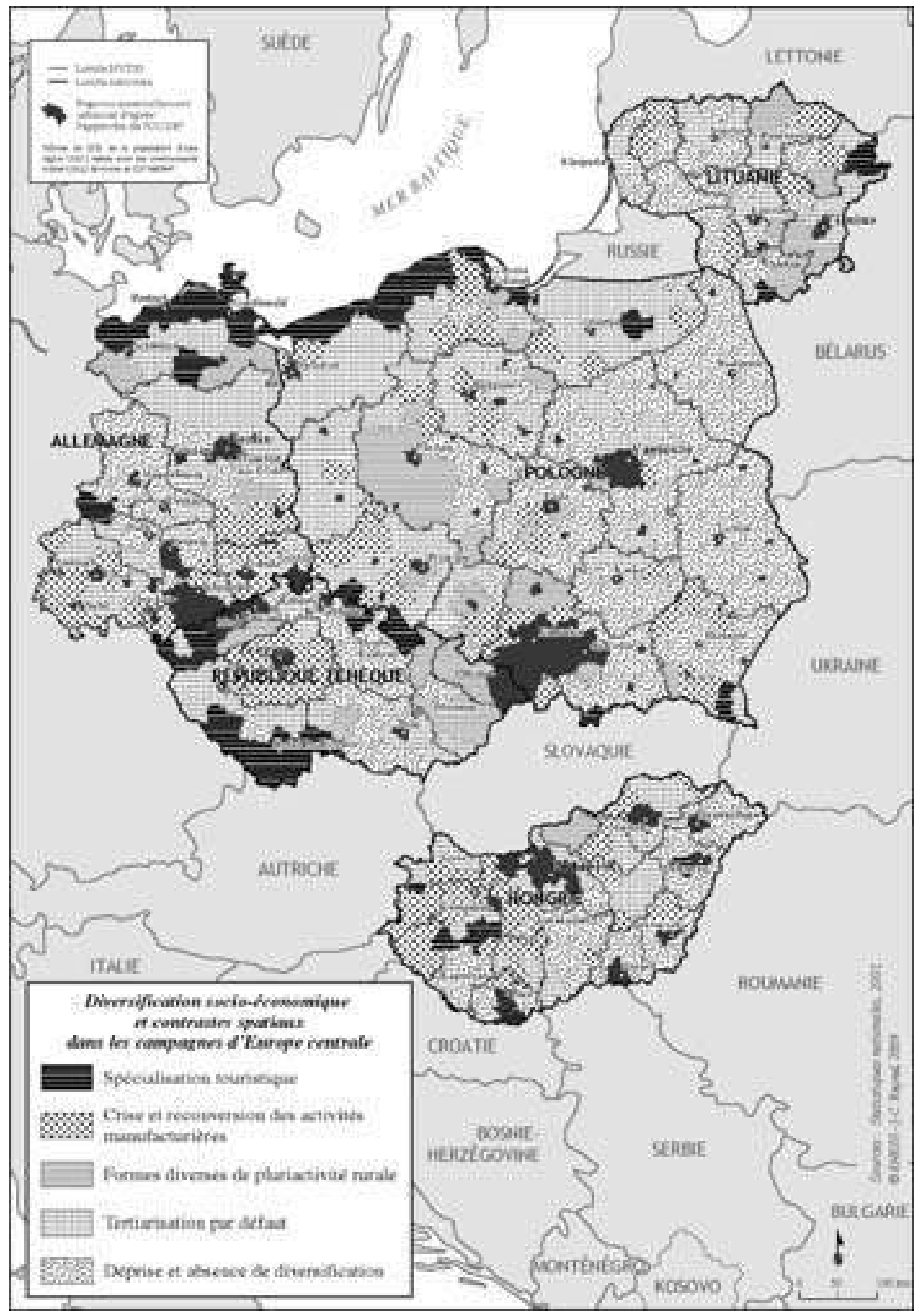


économique et de dépeuplement. Mais dans la plupart des espaces ruraux, les processus de diversification se limitent à la tertiarisation des économies locales. Soit la mobilité sectorielle de la main d'œuvre est favorisée par les effets induits de l'extension résidentielle dans les espaces sous influence urbaine, soit elle traduit l'importance relative des emplois de services d'accompagnement ou de substitution dans les espaces de faible densité et sous faible influence urbaine. Cette « tertiarisation par défaut » est le corollaire d'un recul accéléré de l'emploi agricole. En l'absence d'alternatives, le maintien des services publics assure un niveau minimum d'emplois et un encadrement par des équipements de base (type 4, tertiarisation par défaut).

Enfin d'autres territoires ruraux semblent ignorés par les dynamiques d'intégration socio-économique en cours, que ce soit par la spécialisation des activités ou la diversification des ressources. L'inertie des fonctions et l'absence de stratégie de développement local accentuent la marginalisation de territoires dévitalisés et en attente de projet (type 5, déprise et absence de diversification). Ce processus affecte de vastes espaces ruraux, notamment en Pologne orientale, en Lituanie, dans la Grande plaine hongroise ainsi que dans les nouveaux Länder allemands (Maciulyté, 2004, Depraz, 2005, Hirschhausen, Lacquement, 2007, 2008).

Des effets de contextes nationaux et régionaux pèsent sur l'ensemble des dynamiques à l'œuvre, en particulier sur l'essor de formes nouvelles de multifonctionnalité rurale. Les contrastes de densité et de niveau de vie, l'inégale distribution des aménités rurales, les caractères différenciés des structures agraires et de leurs évolutions récentes, les pratiques composites et parfois inabouties de la démocratie locale fondent des trajectoires territoriales qui rendent plus complexes les processus d'adaptation et de restructuration. L'essai typologique n'ignore ni les contrastes régionaux, ni la variété des situations locales, qui relèvent autant de la diversité des structures territoriales héritées, que du degré et du rythme de la transformation.

Les dimensions territoriales de la transformation postsocialiste concernent l'ensemble des activités économiques et se traduisent par des dynamiques sociodémographiques différenciées. Loin de s'effacer, les disparités héritées ont eu tendance à se renforcer sous l'effet des changements structurels rapides et profonds liés au passage à l'économie de marché. L'essai de typologie ci-dessus a souligné les modalités de diversification des espaces ruraux en distinguant les principales dominantes fonctionnelles. Celles-ci s'ordonnent selon un gradient de spécialisation-intégration, des espaces monofonctionnels aux espaces associant fonctions et usages multiples.

\section{Trajectoires de recomposition des territoires ruraux}

L'ampleur des restructurations observées atteste des efforts poursuivis par les acteurs du monde rural en vue d'une adaptation. En même temps, les recompositions progressent de manière extrêmement inégale selon les pays et les régions, en fonction du poids des héritages structurels, de la disparité des potentiels de transformation et des trajectoires suivies. L'analyse du changement, envisagé dans son caractère multidimensionnel, relève d'une grille de lecture à la fois diachronique et synchronique qui implique de prendre en compte l'interaction entre processus de recomposition et de différenciation. Aux diverses échelles spatiales, les trajectoires procèdent d'un effet de temps et d'un effet de lieu qui renvoient aux durées variables des structures spatiales. Ici, elles perdent leur fonctionnalité et leurs traces s'effacent, là elles perdurent et s'adaptent à la nouvelle donne. 


\section{Dynamiques polymorphes d'intégration socio-économique}

La transformation des structures économiques a impliqué les sociétés locales dans un processus d'intégration procédant d'impulsions à la fois internes et externes. D'une part, la reconfiguration fonctionnelle des territoires ruraux s'appuie sur les capacités endogènes de mobilisation, d'organisation et d'investissement des acteurs sociaux. D'autre part, le processus s'inscrit dans la dépendance de pôles externes de commandement économique. Leurs effets sur la structuration des territoires peuvent être décrits en fonction des modalités de leur intégration socio-économique.

\section{L'intégration sous influence ou sous dépendance}

La métropolisation et l'étalement périurbain transforment les fonctions des territoires ruraux dans le cadre d'un processus exogène d'induction économique. La proximité urbaine favorise une intégration sous influence qui caractérise la périphérie des principales agglomérations. Les formes de dépendance ne se limitent pas aux espaces périurbains. La réorganisation des filières agroalimentaires (à l'initiative des multinationales) place sous tutelle la plupart des espaces à vocation agricole. Elle conduit à la formation de bassins spécialisés de production et à la concentration des structures d'exploitation. Elle dirige les investissements vers le capital d'exploitation et accélère le délestage de main-d'œuvre agricole. La rente naturelle et le fonctionnement du marché contingenté de la PAC orientent les systèmes de cultures, tandis que les objectifs de productivité déterminent la nature et le fonctionnement du système d'exploitation ${ }^{8}$. Ce type d'exploitation des ressources agricoles ne s'accompagne pas

8. Pour une présentation détaillée on se reportera au chapitre VII, de l'ouvrage «Agriculture et ruralité en Europe centrale » p. 140-143 (Maurel et Lacquement, 2007). systématiquement de la prospérité rurale, les effets d'entraînement sur les autres activités rurales demeurant limités. Elle privilégie une forme exogène d'intégration, certes performante, mais qui reste dépendante et extravertie.

\section{L'intégration par la valorisation des héritages structurels}

En dehors des zones périurbanisées, les situations de prospérité rurale, même relative, ou de crise modérée relèvent plutôt de la reconversion et de la valorisation des héritages structurels. Les fonctions spatiales s'appuient alors sur les structures économiques en place dont l'adaptation peut conduire soit à une spécialisation, soit à une diversification des activités. La concentration de la fréquentation touristique au bénéfice des littoraux ou des massifs montagneux conforte la spécialisation d'un certain nombre d'espaces ruraux. À la faveur de la modernisation des infrastructures d'hébergement et de loisirs, l'activité touristique implique les collectivités locales dans des projets de rénovation, de réhabilitation et de développement qui induisent emplois permanents ou saisonniers, et revenus principaux ou complémentaires.

La diversification fonctionnelle mobilise plusieurs secteurs d'activité et s'accompagne d'un recyclage des savoir-faire de la population active. Les exploitations agricoles tentent de compenser la médiocrité de la rente naturelle en développant les activités de valorisation de la production agricole (transformation agroalimentaire et vente directe, agritourisme), mais aussi en participant aux programmes agro-environnementaux de préservation des milieux naturels et de conservation des paysages. Le tourisme vert se reconfigure à partir du réseau traditionnel des sites remarquables ou des stations de cure, et se diffuse également sur la base de l'identification de ressources composées en patrimoine rural (paysages agraires, bâti religieux, historique ou villageois, fêtes et traditions 
locales). Les savoir-faire industriels peuvent attirer les investisseurs (nationaux et étrangers) qui promeuvent la diffusion d'ateliers de production dans le cadre de $\mathrm{PME}^{9}$. Dynamisée par les impulsions endogènes comme par les investissements externes, cette multifonctionnalité rurale soutient l'activité économique et procure des revenus à la population locale.

\section{Marginalisation fonctionnelle et faible intégration}

La dynamique d'intégration semble ignorer une partie des espaces ruraux demeurés à l'écart des principaux flux d'investissement. La crise a durement frappé les structures agraires héritées du socialisme et leur restructuration piétine. Le dépérissement des activités entraîne le repli sur la petite exploitation non marchande et la pratique d'une agriculture de subsistance. Ces espaces ont pour principale fonction de constituer un réservoir de populations migrantes (collines de Baltijos en Lituanie, Pologne du sud-est, Hongrie orientale). La réduction de la charge d'actifs agricoles à l'hectare n'est pas nécessairement le signe d'une meilleure intégration socio-économique. Les structures post-socialistes ne sont que faiblement transformées par les impulsions externes pour promouvoir l'insertion de l'économie locale dans les réseaux économiques régionaux et nationaux. Le sort des populations locales est alors suspendu aux mesures des programmes d'aide publique. C'est en particulier le cas, en Hongrie et en Slovaquie, des villages peuplés par la minorité rom où sévissent chômage et grande pauvreté.

9. Voir les recherches monographiques sur la microrégion de Bóly, en Transdanubie méridionale (Maurel et Póla, 2010).

\section{Une ruralité recomposée}

L'orientation du profil d'activités, l'inégale intensité de la crise et le mode d'intégration socio-économique conjuguent leurs effets pour dessiner une structure spatiale en mosaïque. Les logiques d'organisation dépendent tout d'abord des héritages structurels qui se différencient à l'échelle régionale. Les territoires locaux portent l'empreinte de structures sociales et économiques inscrites et/ou stratifiées dans des temporalités qui semblent ici rejouer dans le processus de transformation post-socialiste. La ruralité centre-européenne se recompose sur les formes spatiales héritées des systèmes antérieurs. Plus précisément, elle se construit sur des itinéraires de spécialisation et de diversification fonctionnelle qui prennent leur accroche sur les formes d'organisation spatiale et de structuration sociale propres à chaque territoire. La dynamique contemporaine est par ailleurs singulièrement polarisée par une série d'effets de lieux. La proximité urbaine joue un rôle décisif, car elle accélère le rythme et amplifie la portée de la transformation. La concentration des emplois dans les villes attire ceux qui cherchent du travail, les marchés urbains drainent les produits locaux et alimentent les flux de tourisme et de loisirs, enfin l'économie urbaine et l'étalement résidentiel accompagnent la migration de l'activité et des emplois induits en périphérie.

De la même façon, la proximité de la frontière intensifie les flux de toutes sortes : migrations pendulaires de travail, flux de capitaux et d'investissements dans une logique de sous-traitance, flux de marchandises et fréquentation touristique. Enfin, les caractères physiques des lieux déterminent la valeur de la rente naturelle et le mode d'exploitation des ressources rurales au regard des perspectives de profit et de rentabilité économique : le potentiel agronomique des sols oriente la spécialisation des systèmes de cultures et la qualité des formes paysagères font varier le niveau d'attractivité touristique. 
Figure 1. Essai de typologie du rural centre-européen

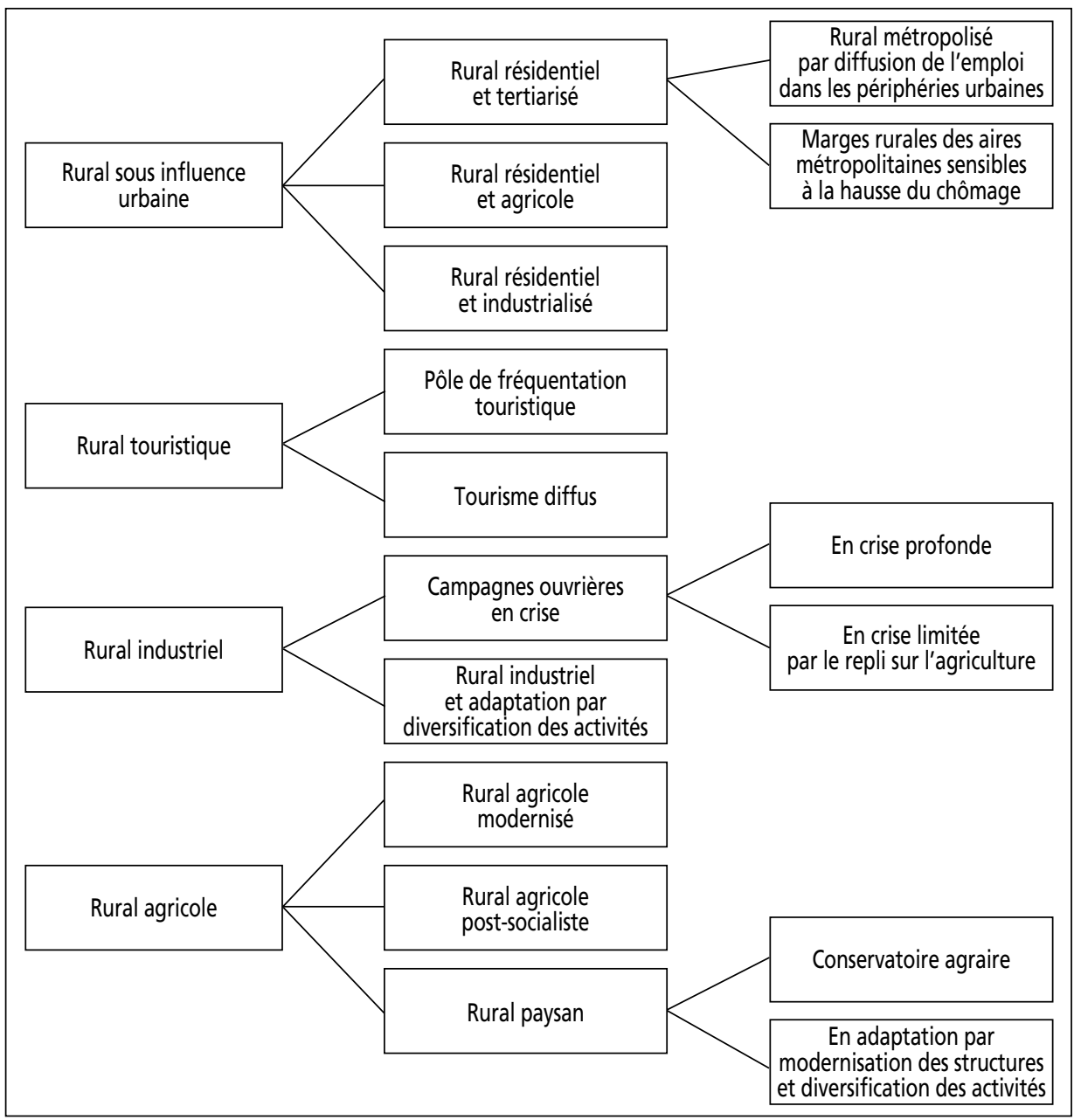

Source : les auteurs

La ruralité centre-européenne se compose de multiples facettes dont l'agencement produit quatre grands types de structuration territoriale (figure 1). Les essais typologiques ont mis en évidence des processus sociétaux communs aux espaces ruraux des anciens et des nouveaux pays membres qui renvoient à l'évolution des activités et des modes de vie dans les sociétés urbanisées. Avec un temps de retard, les trajectoires rurales centre-européennes témoignent de tendances similaires. La remarque est moins simple qu'il n'y paraît. En réalité, le décalage temporel affectant les transformations des campagnes de l'Europe centrale par rapport à celles de l'Europe occidentale a valeur de rupture. L'ancien régime communiste affectait des finalités productives aux espaces ruraux, au service d'un projet politique et social rapprochant la ville et la campagne. Avec un temps de retard par rapport à l'Europe occidentale, l'économie rurale des pays d'Europe centrale s'est émancipée de la domination de l'activité agricole. Auparavant intégrées au sein des structures collectivistes, les activités économiques et sociales, désolidarisées les unes des autres, se sont réorganisées dans le cadre d'entreprises privées de taille diverse. Les fonctions assignées aux territoires ruraux 
dans le nouveau modèle de croissance, adopté par les économies centre-européennes, ont été redéfinies. La disparition d'un monde rural monofonctionnel (à dominante agricole) a laissé place à un rural multifonctionnel et polymorphe.

Les reconfigurations reposent sur la mobilisation de nouvelles ressources. Aux activités productives, agricole et non agricole, sont venues s'ajouter les fonctions résidentielle et récréative, ainsi que les fonctions écologique (préservation des espaces de nature) et culturelle (conservation du patrimoine rural, matériel et immatériel). L'espace rural est devenu le support d'usages diversifiés. Le ré-agencement de ces usages qui mobilisent les ressources sur un même territoire peut être source de tensions. L'arbitrage entre des usages économiques et non économiques se révèle d'autant plus délicat qu'il fait intervenir une pluralité de formes d'appropriation des ressources et des biens impliquant des personnes privées et des personnes publiques. L'identification des biens ruraux est devenue plus difficile, les biens privés (terres agricoles, forêts, lacs et plans d'eau, etc.) ayant en même temps le statut de biens de nature, c'est-à-dire de biens communs. De fait, la multifonctionnalité de l'espace rural rend sa gestion publique plus complexe parce qu'elle fait intervenir un plus grand nombre d'acteurs sociaux, des propriétaires, des producteurs, des résidents permanents ou temporaires, des usagers ainsi que les représentants de ces diverses catégories. Cette situation, en soi radicalement nouvelle, exige non seulement un changement de normes mais également une adaptation des comportements et des pratiques des différentes catégories d'utilisateurs afin de réguler les tensions et de prévenir les conflits d'usage. Elle invite à développer de nouvelles formes de concertation et de participation dont la mise en œuvre s'accomplit à l'échelon local. Les premiers chocs essuyés, les collectivités locales ont entrepris de reformuler leur projet de développement, en même temps qu'elles expérimentaient de nouvelles modalités d'articulation avec la société globale.

\section{Conclusion}

Le paradigme de la transition, utile pour analyser les étapes du passage à l'économie de marché et les réformes institutionnelles du changement de régime, montre ses limites pour lire et interpréter les processus de la transformation et ses effets sur les territoires. Il tend à minimiser le rôle des héritages du passé ou ne le conçoit qu'en termes d'inertie et de blocages s'opposant à la diffusion du nouveau système. Or, si la transition de système est à l'origine du changement des fonctions et des usages des espaces ruraux, elle n'en détermine pas les mécanismes qui font place aux capacités d'adaptation et d'anticipation des acteurs sociaux. La transformation des campagnes décollectivisées a donné naissance à des structures socioéconomiques hybrides et à des formes spatiales composites. Selon les lieux, le rythme et les modalités d'intégration de ces structures postsocialistes au système global diffèrent. Pour interpréter les dynamiques en cours, les nouveaux profils d'activité et les modalités de recomposition des territoires, l'analyse géographique a préféré mobiliser le concept de trajectoire, parce qu'il appréhende les évolutions territoriales en interrogeant la dépendance au passé et le contexte géographique de réception du changement par les sociétés locales $^{10}$. Le changement des territoires ruraux n'est ni immédiat ni univoque, il donne naissance à des reconfigurations polymorphes.

10. Dans le processus de recomposition à l'œuvre, les configurations historiques sont à l'origine d'un « chemin de dépendance » (Maurel, 2009). 


\section{RÉFÉRENCES BIBLIOGRAPHIQUES}

Bański J., Stola W. (2002). Transformation of the Spatial and Functional Structure of Rural Areas in Poland. Studia Obszarów Wiejskich, vol. 3, 13 p.

Chevalier P. (2008). Diversification et mutation des bases économiques dans l'espace rural tchèque. Revue d'études comparatives Est-Ouest, vol. 39, $\mathrm{n}^{\circ} 4$, décembre, p. 143-164.

Csatári B. (2005), "Criteria of Rurality for the Hungarian Micro-regions; Major problems facing Rural Areas in Hungary", in Barta (Györgyi), Fekete (Eva), Kukorelli (Irén) et Timár (Judit), Hungarian Spaces and Places: Patterns of Transition, Centre for Regional Studies, Pécs, 2005, p. 466-482.

Depraz S. (2005). Développement local et politiques de gestion des communes rurales en Hongrie centrale. Bulletin de l'Association de géographes français, juin, p. 168-181.

Hann C. (2003). The Post-Socialist Agrarian Question: Property Relations and the Rural Condition. Lit, Münster, 472 p.

Gorlach K., Kovach I., (2006). Land Use, Nature Conservation and Biodiversity in Central Europe (the Czech, Hungarian and polish Cases). Working Papers, Corason, (5).

Granberg L., Kovách I., Tovey H. (2001). Europe's Green Ring. Ashgate, Aldershot, $366 \mathrm{p}$.

Hirschhausen B., Lacquement G. (2007). Modèles de l'Ouest, territoires de l'Est. Le développement local et la valorisation du patrimoine dans les campagnes d'Allemagne orientale. Revue d'études comparatives Est-Ouest, vol. 38, $\mathrm{n}^{\circ} 3$, septembre, p. 5-30.

Hirschhausen B., Lacquement G. (2008). De la décollectivisation au développement local en Europe centrale et orientale : les effets d'un changement de paradigme, avant-propos. Revue d'études comparatives Est-Ouest, vol. 39, $\mathrm{n}^{\circ} 4$, décembre, p. 5-12.

Kovach I. (2000). LEADER, a New Social Order and the Central-and East-European Countries. Sociologia Ruralis, vol. 40, n², p. 181-189.

Kovach I. (2002). Leadership, Local Power and Rural Restructuring in Hungary. In Halfacree K., Kovach I., Woodward R. (eds.), "Leadership, Local Power and Rural Restructuring in Contemporary Europe", Aldershot, Burlington, Singapore, Sydney: Ashgate.

Kovach I., Kučerova E. (2006). The Project Class in Central Europe: The Czech and Hungarian Cases. Sociologia Ruralis, vol. 46, $\mathrm{n}^{\circ} 1$, p. 3-19.

Leonard P., Kaneff D. (2002). Post-Socialist Peasant? Palgrave, New York.

Maciulytė J. (2004). La recomposition de l'espace rural lituanien dans la perspective de l'intégration européenne. In « Annales de Géographie », $\mathrm{n}^{\circ}$ 636, mars-avril, p. 188-210.

Marty P., Devaux S. (2009). Social Movements and Public Action: Lessons from Environmental Issues. Prague, Éditions du CEFRES, $193 \mathrm{p}$.

Maurel M.-C., (1994). La transition postcollectiviste, mutations agraires en Europe centrale. Paris, L'Harmattan, 366 p.

Maurel M.-C., Halamska M., Lamarche H. (2004). Le repli paysan. Trajectoires de l'après communisme en Pologne. Paris, L'Harmattan, $252 \mathrm{p}$.

Maurel M.-C., Lacquement G. (2007). Agriculture et ruralité en Europe centrale. Paris, Aux lieux d'être-CEFRES, Atlas, $150 \mathrm{p}$.

Maurel M.-C. (2008). L'action publique « par le bas » : l'approche LEADER en Europe centrale. Revue d'études comparatives Est-Ouest, vol. 39, $\mathrm{n}^{\circ} 4$, décembre, p. 33-61.

Maurel M.-C. (2009). Penser l'historicité 
des territoires. In Chevalier P., Cortès G., Dedeire M., « Héritages et trajectoires rurales en Europe », Paris, L'Harmattan, p. 21-40.

Maurel M.-C., Pola P. (2010). L'émergence du développement local : le cas de Bóly en Transdanubie méridionale. In Halamska M., Maurel M.-C., «Les acteurs locaux à l'épreuve du modèle européen LEADER », CEFRES-IRWIR PAN, Prague, p. 149-184.

Rey V. (1996). Les nouvelles campagnes de l'Europe centre-orientale. CNRS éditions, Paris, $240 \mathrm{p}$.

Rösener W. (1994). Les paysans dans l'histoire de l'Europe. Paris, Éditions du Seuil.
Small (L.-A.) (2003). The Opportunity of Subsistence Style Agriculture in Central Eastern Europe. In Eastern European Countryside, n ${ }^{\circ}$ 9, p. 45-62.

Streith (M.) (2005). Dynamiques paysannes en Mecklembourg, survie d'un savoirfaire. LIT Verlag, coll. Freiburger Sozialanthropologische Studien, vol. 7 , Münster/Hambourg, 232 p.

Swain N. (1999), “Agricultural Restitution and Co-operative Transformation in the Czech Republik, Hungary and Slovakia", Europe-Asia Studies, 51, 1199-1219.

Szúcs J. (1985). Les trois Europe. Paris, L'Harmattan, $128 \mathrm{p}$. 